\title{
Measuring Permissivity in Finite Games
}

Patricia Bouyer, Marie Duflot, Nicolas Markey and Gabriel Renault

Research report LSV-09-12

June 2009

Laboratoire

Spécification

Vérification

CIIS École Normale Supérieure de Cachan

61, avenue du Président Wilson

94235 Cachan Cedex France 



\title{
Measuring Permissivity in Finite Games
}

\author{
Patricia Bouyer $^{1 \star}$, Marie Duflot ${ }^{2}$, Nicolas Markey $^{1 \star}$, and Gabriel Renault ${ }^{3}$ \\ 1 LSV, CNRS \& ENS Cachan, France \\ \{bouyer, markey\}@lsv.ens-cachan.fr \\ 2 LACL, Université Paris 12, France \\ duflot@univ-paris12.fr \\ 3 Département Informatique, ENS Lyon, France \\ gabriel.renault@ens-lyon.fr
}

\begin{abstract}
In this paper, we extend the classical notion of strategies in turn-based finite games by allowing several moves to be selected. We define and study a quantitative measure for permissivity of such strategies by assigning penalties when blocking transitions. We prove that for reachability objectives, most permissive strategies exist, can be chosen memoryless, and can be computed in polynomial time, while it is in NP $\cap$ coNP for discounted and mean penalties.
\end{abstract}

\section{Introduction}

Finite games. Finite games have found numerous applications in computer science [Tho02]. They extend finite automata with several players interacting on the sequence of transitions being fired. This provides a convenient way for reasoning about open systems (subject to uncontrollable actions of their environment), and for verifying their correctness. In that setting, correctness generally means the existence of a controller under which the system always behaves according to a given specification. A controller, in that terminology, is nothing but a strategy in the corresponding game, played on the automaton of the system, against the environment.

Our framework. In this paper, we propose a new framework for computing permissive controllers in finite-state systems. We assume the framework of twoplayer turn-based games (where the players are Player $\diamond$ and Player $\square$, with the controller corresponding to Player $\diamond$ ). The classical notion of (deterministic) strategy in finite (turn-based) games is extended into the notion of multi-strategy, which allows several edges to be enabled. The permissivity of such a multi-strategy is then measured by associating penalties to blocking edges (each edge may have a different penalty). A strategy is more permissive than an other one if its penalty is weaker, i.e., if it blocks fewer (or less expensive) edges.

We focus on reachability objectives for the controller, that is, the first aim of Player $\diamond$ will be to reach a designated set of winning states (whatever

\footnotetext{
* These authors were partly supported by the French project DOTS (ANR-06-SETI003), by the European project QUASIMODO (FP7-ICT-STREP- 214755), and by the ESF project GASICS.
} 
Player $\square$ does). The second aim of Player $\diamond$ will be to minimize the penalty assigned to the set of outcomes generated by the multi-strategy.

Formally we consider weighted (finite) games, which are turn-based finite games with non-negative weights on edges. In each state, the penalty assigned to a multi-strategy is the sum of the weights of the edges blocked by the multi-strategy. Several ways of measuring the penalty of a strategy can then be considered: in this paper, we consider three ways of counting penalties along outcomes (sum, discounted sum, and mean value) and then set the penalty of a multi-strategy as the maximal penalty of its outcomes.

We will be interested in several problems: $(i)$ does Player $\diamond$ have a winning multi-strategy for which the penalty is no more than a given threshold? (ii) compute the infimal penalty that Player $\diamond$ can ensure while reaching her goal; (iii) synthesize (almost-)optimal winning multi-strategies, and characterize them (in terms of memory and regularity).

Our results. We first prove that our games with penalties can be transformed into classical weighted games [ZP96,LMO06] with an exponential blowup, and that the converse reduction is polynomial.

Then, we prove that we can compute optimal and memoryless multi-strategies for optimal reachability in PTIME. The proof is in three steps: first, using our transformation to weighted games and results of [LMO06], we obtain the existence of optimal memoryless multi-strategies; we then propose a polynomialtime algorithm for computing an optimal winning multi-strategy with memory; finally, we show how we can get rid of the memory in such a multi-strategy, which yields the expected result.

We then focus on two other ways of computing penalties, namely the discounted sum and the mean value, and we prove that optimal multi-strategies may not exist, or may require memory. We further prove that we can compute the optimal discounted penalty in NP $\cap$ coNP, and that we can search for almostoptimal winning multi-strategies as a pair $\left(\sigma_{1}, \sigma_{2}\right)$ of memoryless multi-strategies and that we need to play $\sigma_{1}$ for some time before following $\sigma_{2}$ in order to reach the goal. The longer we play $\sigma_{1}$, the closer we end up to the optimal discounted penalty. The same holds for the mean penalty before reaching the goal.

As side-results, we obtain the complexity of computing strategies in weighted games with a combined objective of reaching a goal state and optimizing the accumulated cost. This can be seen as the game version of the shortest path problem in weighted automata. Regarding accumulated costs, this was already a by-product of [LMO06]; we show here that for discounted and mean costs, optimal or memoryless optimal strategies do not necessarily exist, but almost-optimal strategies can be obtained as a "pair" of memoryless strategies.

Related work. This quantitative approach to permissivity is rather original, and does not compare to either of the approaches found in the literature [BJW02,PR05]. Indeed classical notions of permissivity imply the largest sets of generated plays. This is not the case here, where an early cut of a branch/edge of the game may avoid a large penalty later on for blocking many edges. However our notion 
of multi-strategy coincides with the non-deterministic strategies of [BJW02] and [Lut08].

Our work also meets the problem proposed in [CHJ05] of considering mixed winning objectives, one which is qualitative (parity in their special case), and one which is quantitative (mean-payoff in their special case). The same kind of mixed objectives is considered when extending ATL with quantitative constraints [LMO06,HP06].

The rest of this paper is organized as follows. In the next section, we introduce our formalism of multi-strategies and penalties. We also explain the link with the classical framework of games with costs. Section 3 is devoted to our polynomialtime algorithm for computing most permissive strategies. Section 4 deals with the case of discounted and mean penalty. By lack of space, several proofs have been postponed to the technical appendix.

\section{Weighted Games with Reachability Objectives}

\subsection{Basic definitions}

Weighted games. A (finite) weighted game is a tuple $\mathrm{G}=\left(V_{\square}, V_{\diamond}, E\right.$, weight) where $V_{\square}$ and $V_{\diamond}$ are finite sets of states (said to belong to Player $\square$ and Player $\diamond$, resp.); writing $V=V_{\square} \cup V_{\diamond} \cup\{\odot, \odot\}$ where $\odot$ and $\odot$ are two distinguished states not belonging to $V_{\square} \cup V_{\diamond}, E \subseteq V \times V$ is a finite set of edges; and weight: $E \rightarrow \mathbb{N}$ is a function assigning a weight to every edge. We assume (w.l.o.g.) that the states $(-)$ and $(;)$ have no outgoing edges (they are respectively the winning and losing states). If $v \in V$, we write $v E$ (resp. $E v)$ for $E \cap(\{v\} \times V)($ resp. $E \cap(V \times\{v\}))$ for the set of edges originating from (resp. targetting to) $v$.

A run $\varrho$ in $\mathrm{G}$ is a finite or infinite sequence of states $\left(v_{i}\right)_{0 \leq i \leq p}$ (for some $p \in \mathbb{N} \cup\{\infty\})$ such that $e_{i}=\left(v_{i-1}, v_{i}\right) \in E$ when $0<i \leq p$. We may also write for such a run $\varrho=\left(v_{0} \rightarrow v_{1} \rightarrow v_{2} \cdots\right)$, or $\varrho=\left(e_{i}\right)_{i \geq 1}{ }^{4}$, or the word $\varrho=v_{0} v_{1} v_{2} \ldots$ The length of $\varrho$, denoted by $|\varrho|$, is $p+1$. For finite-length runs, we write last $(\varrho)$ for the last state $v_{p}$. Given $r<|\varrho|$, the $r$-th prefix of $\varrho=\left(v_{i}\right)_{0 \leq i \leq p}$ is the run $\varrho_{\leq r}=\left(v_{i}\right)_{0 \leq i \leq r}$. Given a finite run $\varrho=\left(v_{i}\right)_{0 \leq i \leq p}$ and a transition $e=\left(v, v^{\prime}\right)$ with $v=v_{p}$, we write $\varrho \stackrel{e}{\rightarrow}$, or $\varrho \rightarrow v^{\prime}$, for the run $\varrho=\left(v_{i}\right)_{0 \leq i \leq p+1}$ with $v_{p+1}=v^{\prime}$.

We write Runs $s_{G}^{<\omega}$ (resp. Runs $s_{G}^{\omega}$ ) for the set of finite (resp. infinite) runs in G, and Runs $_{G}=\operatorname{Runs}_{G}^{<\omega} \cup \operatorname{Runs}_{G}^{\omega}$. In the sequel, we omit the subscript $G$ when no ambiguity may arise.

Multi-strategies. A multi-strategy for Player $\diamond$ is a function

$$
\sigma:\left\{\varrho \in \operatorname{Runs}^{<\omega} \mid \operatorname{last}(\varrho) \in V_{\diamond}\right\} \rightarrow 2^{E}
$$

such that, for all $\varrho \in$ Runs $^{<\omega}$, we have $\sigma(\varrho) \subseteq v E$ with $v=$ last $(\varrho)$. A multistrategy is memoryless if $\sigma(\varrho)=\sigma\left(\varrho^{\prime}\right)$ as soon as last $(\varrho)=\operatorname{last}\left(\varrho^{\prime}\right)$. A memoryless

\footnotetext{
${ }^{4}$ These notations are equivalent since we assume that there can only be one edge between two states.
} 
multi-strategy $\sigma$ can be equivalently represented as a mapping $\sigma^{\prime}: V_{\diamond} \rightarrow 2^{E}$, with $\sigma(\varrho)=\sigma^{\prime}(\operatorname{last}(\varrho))$.

Multi-strategies extend the usual notion of strategies by selecting several possible moves (classically, a strategy is a multi-strategy $\sigma$ such that for every $\varrho \in \operatorname{Runs}^{<\omega}$ with last $(\varrho) \in V_{\diamond}$, the set $\sigma(\varrho)$ is a singleton). The aim of this paper is to compare multi-strategies and to define and study a quantitative notion of permissivity of a multi-strategy.

Given a multi-strategy $\sigma$ for Player $\diamond$, the set of outcomes of $\sigma$, denoted Out $(\sigma) \subseteq$ Runs, is defined as follows:

- for every state $v \in V$, the run $v$ is in Out ${ }^{<\omega}(\sigma)$;

- if $\varrho \in$ Out $^{<\omega}(\sigma)$ and $\sigma(\varrho)$ is defined and non-empty, then for every $e \in \sigma(\varrho)$, the run $\varrho \stackrel{e}{\rightarrow}$ is in Out ${ }^{<\omega}(\sigma)$;

- if $\varrho \in$ Out $^{<\omega}(\sigma)$ and last $(\varrho)=v \in V_{\square}$, then for every edge $e \in v E$, the run $\varrho \stackrel{e}{\rightarrow}$ is in $\mathrm{Out}^{<\omega}(\sigma)$;

- if $\varrho \in$ Runs $^{\omega}$ and if all finite prefixes $\varrho^{\prime}$ of $\varrho$ are in Out ${ }^{<\omega}(\sigma)$, then $\varrho \in \operatorname{Out}^{\omega}(\sigma)$.

We write Out $(\sigma)=\operatorname{Out}^{<\omega}(\sigma) \cup \operatorname{Out}^{\omega}(\sigma)$. A run $\varrho$ in Out $(\sigma)$ is maximal whenever it is infinite, or it is finite and either $\sigma(\varrho)=\varnothing$, or last $(\varrho)$ has no outgoing edge (i.e., the set $v E$, with $v=\operatorname{last}(\varrho)$, is empty). If $\varrho_{0}$ is a finite outcome of $\sigma$, we write $\operatorname{Out}\left(\sigma, \varrho_{0}\right)$ (resp. Out $\left.{ }^{\max }\left(\sigma, \varrho_{0}\right)\right)$ for the set of outcomes (resp. maximal outcomes) of $\sigma$ having $\varrho_{0}$ as a prefix. A multi-strategy $\sigma$ is winning after $\varrho_{0}$ if every run $\varrho \in \operatorname{Out}^{\max }\left(\sigma, \varrho_{0}\right)$ is finite and has last $(\varrho)=\odot$. A finite run $\varrho_{0}$ is winning if it admits a winning multi-strategy after $\varrho_{0}$. Last, a strategy is winning if it is winning from any winning state (seen as a finite run).

Penalties for multi-strategies. We define a notion of permissivity of a multistrategy by counting the weight of transitions that the multi-strategy blocks along its outcomes. If $\sigma$ is a multi-strategy and $\varrho_{0}$ is a finite run, the penalty of $\sigma$ after $\varrho_{0}$, denoted penalty $\left(\sigma, \varrho_{0}\right)$, is defined as $\sup \left\{\right.$ penalty $_{\sigma, \varrho_{0}}(\varrho) \mid \varrho \in$ Out $\left.^{\max }\left(\sigma, \varrho_{0}\right)\right\}$ where penalty $_{\sigma, \varrho_{0}}(\varrho)$ is defined inductively, for every finite run $\varrho \in \operatorname{Out}\left(\sigma, \varrho_{0}\right)$, by:

- penalty,$_{\sigma, \varrho_{0}}\left(\varrho_{0}\right)=0$;

- if last $(\varrho) \notin V_{\diamond}$ and $(\operatorname{last}(\varrho), v) \in E$, then penalty,$_{\sigma, \varrho_{0}}(\varrho \rightarrow v)=$ penalty $_{\sigma, \varrho_{0}}(\varrho)$;

- if last $(\varrho) \in V_{\diamond}$ and $(\operatorname{last}(\varrho), v) \in \sigma(\varrho)$, then

$$
\begin{gathered}
\text { penalty }_{\sigma, \varrho_{0}}(\varrho \rightarrow v)=\text { penalty }_{\sigma, \varrho_{0}}(\varrho)+\sum_{\left(\operatorname{last}(\varrho), v^{\prime}\right) \in(E \backslash \sigma(\varrho))} \operatorname{weight}\left(\operatorname{last}(\varrho), v^{\prime}\right) ; \\
\text { - if } \varrho \in \operatorname{Out}\left(\sigma, \varrho_{0}\right) \cap \operatorname{Runs}^{\omega} \text {, then penalty }{ }_{\sigma, \varrho_{0}}(\varrho)=\lim _{n \rightarrow+\infty} \text { penalty }_{\sigma, \varrho_{0}}\left(\varrho_{\leq n}\right) .
\end{gathered}
$$

The first objective of Player $\diamond$ is to win the game (i.e., reach $\odot)$ ), and her second objective is to minimize the penalty. In our formulation of the problem, Player $\square$ has no formal objective, but her aim is to play against Player $\diamond$ (this is a zero-sum game), which implicitly means that Player $\square$ tries to avoid reaching $\odot$, and if this is not possible, she tries to maximize the penalty before reaching $\odot$. 
We write opt_penalty $\left(\varrho_{0}\right)$ for the optimal penalty Player $\diamond$ can ensure after $\varrho_{0}$ while reaching $\odot$ :

$$
\text { opt_penalty }\left(\varrho_{0}\right)=\inf \left\{\text { penalty }\left(\sigma^{\prime}, \varrho_{0}\right) \mid \sigma^{\prime} \text { winning multi-strategy after } \varrho_{0}\right\} \text {. }
$$

It is equal to $+\infty$ if and only if Player $\diamond$ has no winning multi-strategy after $\varrho_{0}$.

The following lemma is rather obvious, and shows that we only need to deal with the optimal penalty from a state.

Lemma 1. Let $\mathrm{G}$ be a weighted game, let $\varrho$ and $\varrho^{\prime}$ be two runs in $\mathrm{G}$ such that $\operatorname{last}(\varrho)=\operatorname{last}\left(\varrho^{\prime}\right)$. Then opt_penalty $(\varrho)=$ opt_penalty $\left(\varrho^{\prime}\right)$.

Given $\varepsilon \geq 0$, a winning multi-strategy $\sigma$ is $\varepsilon$-optimal after $\varrho_{0}$ if penalty $\left(\sigma, \varrho_{0}\right) \leq$ opt_penalty $\left(\varrho_{0}\right)+\varepsilon$. It is optimal after $\varrho_{0}$ when it is 0 -optimal after $\varrho_{0}$. If $\sigma$ is $\varepsilon$-optimal from any winning state, then we say that $\sigma$ is $\varepsilon$-optimal.

Classical weighted games. This way of associating values to runs and (multi-) strategies is rather non-standard, and usually it is rather a notion of accumulated cost along the runs which is considered. It is defined inductively as follows:

$-\operatorname{cost}(v)=0$ for single-state runs;

$-\operatorname{cost}(\varrho \stackrel{e}{\rightarrow})=\operatorname{cost}(\varrho)+$ weight $(e)$ otherwise.

Then again, if $\sigma$ is a multi-strategy and $\varrho_{0}$ is a finite outcome, $\operatorname{cost}\left(\sigma, \varrho_{0}\right)=$ $\sup \left\{\operatorname{cost}(\varrho)-\operatorname{cost}\left(\varrho_{0}\right) \mid \varrho \in\right.$ Out $\left.^{\max }\left(\sigma, \varrho_{0}\right)\right\}$, and notions of $(\varepsilon-)$ optimal strategies are defined in the expected way.

Example 1. A weighted game is depicted on Fig. 1. For this example, it can be easily seen that the optimal strategy w.r.t. costs from state $a$ consists in going through $b$, resulting in a weight of 6 .

Regarding penalties and multi-strategies, the situation is more difficult. From state $b$, there is only one way of winning, with penalty 6 (because the strategy must block the transition to the losing state). From $d$, we have two possible winning multi-strategies: either block the transition to $b$, with penalty 2 , or keep it; in the latter case, we will then have penalty 6 in state $d$, as explained above. In $d$, the best multi-strategy thus

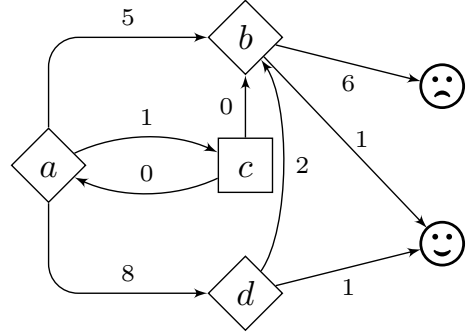

Fig. 1. A weighted game amounts to blocking the transition to $b$, so that we can win with penalty 2 . Now, from $a$, it seems natural to try to go winning via $d$. This requires blocking both transitions to $b$ and $c$, and results in a global penalty of $8(=5+1+2)$ for winning. However, allowing both transitions to $b$ and $d$ is better, as the global (worst case) penalty in this case is $7(=1+6)$. Note that in that case, it is also possible to allow transition to $c$ for some time, since the loop between $a$ and $c$ will add no extra penalty. But if we allow it forever, it will not be winning, this transition to $c$ has thus to be blocked at some point in order to win. 
Computation and decision problems. We let $\mathrm{G}=\left(V_{\square}, V_{\diamond}, E\right.$, weight $)$ be a weighted game. Given $v \in V$, we will be interested in computing the value opt_penalty $(v)$, and if an optimal winning multi-strategy exists, in computing it. We will also be interested in computing for every $\varepsilon>0$, an $\varepsilon$-optimal winning multi-strategy.

Formally, the optimal reachability problem with penalty we consider is the following: given a weighted game $\mathrm{G}$, a rational number $c$ and a state $v \in V$, does there exist a multi-strategy $\sigma$ for Player $\diamond$ such that penalty $(\sigma, v) \leq c$.

\subsection{From penalties to costs, and back}

Penalties and costs assume very different points of view: in particular, costoptimality can obviously be achieved with "deterministic" strategies (adding extra outcomes can only increase the overall cost of the strategy), while penaltyoptimality generally requires multi-strategies. Still, there exists a tight link between both approaches, which we explain on two examples (Figs. 2 and 3).

Lemma 2. For every weighted game $\mathrm{G}=\left(V_{\square}, V_{\diamond}, E\right.$, weight $)$, we can construct an exponential-size weighted game $\mathrm{G}^{\prime}=\left(V_{\square}^{\prime}, V_{\diamond}^{\prime}, E^{\prime}\right.$, weight $\left.{ }^{\prime}\right)$ such that $V_{\square} \subseteq V_{\square}^{\prime}$, $V_{\diamond} \subseteq V_{\diamond}^{\prime}$ and, for any state $v \in V_{\square} \cup V_{\diamond}$ and any bound $c$, Player $\diamond$ has a winning multi-strategy with penalty $c$ from $v$ in $\mathrm{G}$ iff she has a winning strategy with cost $c$ from $v$ in $\mathrm{G}^{\prime}$.
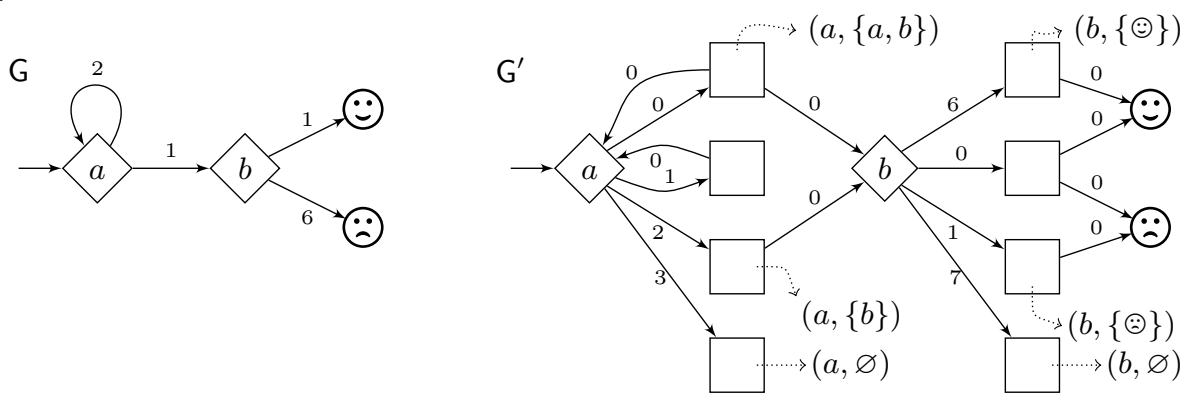

Fig. 2. From penalties (and multi-strategies) to costs (and strategies)

Lemma 3. For every weighted game $\mathrm{G}^{\prime}=\left(V_{\square}^{\prime}, V_{\diamond}^{\prime}, E^{\prime}\right.$, weight $\left.{ }^{\prime}\right)$, we can construct a polynomial-size weighted game $\mathrm{G}=\left(V_{\square}, V_{\diamond}, E\right.$, weight $)$ such that $V_{\square}^{\prime} \subseteq V_{\square}$, $V_{\diamond}^{\prime} \subseteq V_{\diamond}$, and for any state $v \in V_{\square}^{\prime} \cup V_{\diamond}^{\prime}$ and any value $c$, Player $\diamond$ has a winning strategy with cost $c$ from $v$ in $\mathrm{G}^{\prime}$ iff she has a winning multi-strategy with penalty $c$ from $v$ in $\mathrm{G}$.

\section{Optimal Reachability in Penalty Games}

Classical weighted games are known to admit memoryless optimal strategies (see e.g. [LMO06]). Hence, applying Lemma 2 we know that we can solve the optimal reachability problem with penalty in NP: memoryless multi-strategies are 

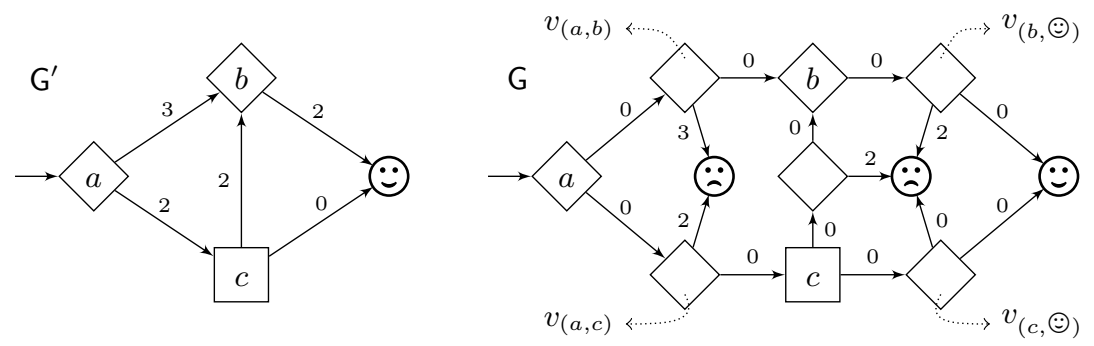

Fig. 3. From costs (and strategies) to penalties (and multi-strategies)

sufficient to win optimally, and we can thus guess a memoryless multi-strategy and check, in polynomial time, that it is winning and has penalty less than the given threshold. This section is devoted to the two-step proof of the following (stronger) result:

Theorem 4. The optimal reachability problem with penalty can be solved in PTIME.

In the sequel, we let $\mathrm{G}=\left(V_{\square}, V_{\diamond}, E\right.$, weight $)$ be a weighted game.

\subsection{Construction of an optimal winning multi-strategy}

In this section, we give a polynomial-time algorithm for computing an optimal winning multi-strategy (which requires memory). The idea is to inductively compute the penalty for winning in $j$ steps, for each $j$ less than the number of states. This will be sufficient as we know that there exists a memoryless optimal multi-strategy, which wins in $|V|$ from the winning states.

Due to the transformation presented in Lemma 2, there is a priori an exponential blowup for computing the best move in one step (because Player $\diamond$ can select any subset of the outgoing edges of the current state, and will choose 'the best' subset), but we will show that choices satisfy some monotonicity property that will help making the best choice in polynomial time.

For any integer $k$, we say that a multi-strategy $\sigma$ is $k$-step if, for every run $\varrho$ of length (strictly) larger than $k$ with last $(\varrho) \in V_{\diamond}$, we have $\sigma(\varrho)=\varnothing$. For instance, a memoryless winning multi-strategy $\sigma^{\prime}$ naturally induces winning multi-strategy (all outcomes of $\sigma^{\prime}$ have length no more than $|V|$ and for all the other (useless) runs we can set $\sigma(\varrho)=\varnothing)$. We say that a state $v$ is winning in $k$ steps if there is a $k$-step multi-strategy which is winning from $v$.

The algorithm will proceed as follows: for every $0 \leq j \leq|V|$, we build a $j$-step multi-strategy $\sigma_{j}$ which will be winning from all states that are winning in $j$ steps, and optimal among all those winning $j$-step multi-strategies. We also compute, for each state $v \in V$, a value $c_{v, j}$ which is either the penalty of strategy $\sigma_{j}$ from $v$ (i.e. penalty $\left(\sigma_{j}, v\right)$ ), or $+\infty$ in case $\sigma_{j}$ is not winning from $v$.

Since we know that memoryless multi-strategies suffice to win optimally, we conclude that there exists a $|V|$-step multi-strategy, which is winning and optimal, and the multi-strategy $\sigma_{|V|}$ which we build will then be optimal and winning. It follows that $c_{v,|V|}$ will be equal to opt_penalty $(v)$. 
When $j=0$, we let $\sigma_{0}(\varrho)=\varnothing$ for any $\varrho$ ending in a $V_{\diamond}$-state. It is the only 0-step multi-strategy, so that it clearly is optimal among these. Clearly, $c_{v, 0}=+\infty$ for all $v \neq \odot, c_{\odot, 0}=0$, and $\odot$ is the only state from which we can win with a 0 -step multi-strategy.

We assume we have built $\sigma_{j}(0 \leq j<|V|)$, and we now define $\sigma_{j+1}$. Let $\varrho=$ $v_{0} \rightarrow v_{1} \rightarrow v_{2} \ldots \rightarrow v_{k}$ be a run ending in $V_{\diamond}$. If $k \geq j+1$, we let $\sigma_{j+1}(\varrho)=\varnothing$. Otherwise, if $k \geq 1$, we let $\sigma_{j+1}\left(v_{0} \rightarrow v_{1} \rightarrow v_{2} \ldots \rightarrow v_{k}\right)=\sigma_{j}\left(v_{1} \rightarrow v_{2} \ldots \rightarrow\right.$ $\left.v_{k}\right)$. Finally, when $k=0$ and $\varrho=v$, we let $\left\{u_{1}, \ldots, u_{p}\right\}$ be the set of successors of $v$, assuming that they are ordered in such a way that $c_{u_{r}, j} \leq c_{u_{s}, j}$ if $r \leq s$. Now, let

$$
f_{v, j+1}: I \subseteq \llbracket 1, p \rrbracket \mapsto \sum_{s \notin I} \operatorname{weight}\left(v, u_{s}\right)+\max _{s \in I} c_{u_{s}, j},
$$

and let $I \neq \varnothing$ be a subset of $\llbracket 1, p \rrbracket$ realizing the minimum of $f_{v, j+1}$ over the non-empty subsets of $\llbracket 1, p \rrbracket$. Assume that there exist two integers $l<m$ in $\llbracket 1, p \rrbracket$ such that $l \notin I$ and $m \in I$. Since $u_{l} \leq u_{m}$, we have

$$
f_{v, j+1}(I \cup\{l\})-f_{v, j+1}(I)=-\operatorname{weight}\left(v, u_{l}\right) .
$$

This entails that $I \cup\{l\}$ is also optimal. By repeating the process, we can prove that there exists an interval $\llbracket 1, q \rrbracket$ realizing the minimum of $f_{v, j+1}$. As a consequence, finding the minimum of $f_{v, j+1}$ can be done in polynomial time (by checking all intervals of the form $\llbracket 1, q \rrbracket)$. We write $T_{v, j+1}$ for a corresponding set of states, whose indices realize the minimum of $f_{v, j+1}$. We then define $\sigma_{j+1}(v)=\left\{\left(v, v^{\prime}\right) \mid\right.$ $\left.v^{\prime} \in T_{v, j+1}\right\}$, and $c_{v, j+1}=f_{v, j+1}\left(T_{v, j+1}\right)$ for all $v \in V_{\diamond}$. It is easy to check that $c_{v, j+1}=$ penalty $\left(\sigma_{j+1}, v\right)$ if $\sigma_{j+1}$ is winning from $v$, and $c_{v, j+1}=+\infty$ otherwise.

We now prove that for every $0 \leq j \leq|V|, \sigma_{j}$ is optimal among all $j$-step winning multi-strategies. Assume that, for some $0 \leq j \leq|V|$, there is a $j$-step multi-strategy $\sigma^{\prime}$ that is winning and strictly better than $\sigma_{j}$ from some winning state $v$. We pick the smallest such index $j$. We must have $j>0$ since $\sigma_{0}$ is optimal among the 0-step multi-strategies. Consider the set of successors $\left\{u_{1}, \ldots, u_{p}\right\}$ of $v$ ordered as above, and let $T$ be the set of indices such that $\sigma^{\prime}(v)=\left\{\left(v, u_{t}\right) \mid t \in T\right\}$. Then after one step, the multi-strategy $\sigma^{\prime}$ is $(j-1)$-step and winning from any $u_{t}$ satisfying $\left(v, u_{t}\right) \in \sigma^{\prime}(v)$, and its penalty is thus not smaller than that of the multi-strategy $\sigma_{j-1}$ (by minimality of $j$, we have penalty $\left(\sigma^{\prime}, v \rightarrow u_{t}\right) \geq c_{u_{t}, j-1}$ ). Hence:

$$
\text { penalty }\left(\sigma^{\prime}, v\right) \geq \sum_{s \notin T} \operatorname{weight}\left(v, u_{s}\right)+\max _{t \in T} c_{u_{t}, j-1}=f_{v, j}(T)
$$

On the other hand, as $\sigma^{\prime}$ is strictly better than $\sigma_{j}$ we must have

$$
\text { penalty }\left(\sigma^{\prime}, v\right)<c_{v, j}=f_{v, j}\left(T_{v, j}\right) \leq f_{v, j}(T)
$$

because $T_{v, j}$ achieves the minimum of $f_{v, j}$. This is a contradiction, and from every state $v$ from which there is a $j$-step winning multi-strategy, $\sigma_{j}$ is winning optimally (in $j$ steps).

As stated earlier, due to the existence of memoryless optimal winning multistrategies, $|V|$-step multi-strategies are sufficient and $\sigma_{|V|}$ is optimal winning. 


\subsection{Deriving a memoryless winning multi-strategy}

In this section we compute, from any winning multi-strategy $\sigma$, a memoryless winning multi-strategy $\sigma^{\prime}$ which has lower penalty for Player $\diamond$. The idea is to represent $\sigma$ as a (finite) forest (it is finite because $\sigma$ is winning) where a node corresponds to a finite outcome, and to select a state $v$ for which $\sigma$ is not memoryless yet. This state should be chosen carefully ${ }^{5}$ so that we will be able to "plug" the subtree (i.e., play the multi-strategy) rooted at some node ending in $v$ at all nodes ending in $v$ while keeping all states winning and while decreasing (or at least leaving unchanged) the penalty of all states. This transformation will be repeated until the multi-strategy is memoryless from all states. That way, if $\sigma$ was originally optimal, then so will $\sigma^{\prime}$ be.

Let $\Sigma$ be a finite alphabet. A $\Sigma$-forest is a tuple $\mathcal{T}=(T, R)$ where $T \subseteq \Sigma^{+}$ is a set of non-empty finite words on $\Sigma$ (called nodes) such that, for each $t \cdot a \in T$ with $a \in \Sigma$ and $t \in \Sigma^{+}$, it holds $t \in T$ ( $T$ is closed by non-empty prefix) ; $R \subseteq \Sigma \cap T$ is the set of roots. Given $a \in \Sigma$, a node $t$ such that $t=u \cdot a$ is called an occurrence of $a$. Given a node $t \in T$, the depth of $t$ is $|t|-1$ (where $|t|$ is the length of $t$ seen as a word on $\Sigma$ ), and its height, denoted height $t_{\mathcal{T}}(t)$, is

$$
\sup \left\{|u| \mid u \in \Sigma^{*} \text { and } t \cdot u \in T\right\} .
$$

In particular, height $\mathcal{T}_{\mathcal{T}}(t)=+\infty$ when $t$ is the prefix of an infinite branch in $\mathcal{T}$.

A $\Sigma$-tree is a $\Sigma$-forest with one single root. Given a forest $\mathcal{T}=(T, R)$ and a node $t \in T$, the subtree of $\mathcal{T}$ rooted at $t$ is the tree $\mathcal{S}=(S,\{n\})$ where $n=\operatorname{last}(t)$ and $s \in S$ iff $t \cdot s \in T$.

Let $\mathrm{G}=\left(V_{\square}, V_{\diamond}, E\right.$, weight) be a weighted game. A winning multi-strategy $\sigma$ for Player $\diamond$ in $G$ and a winning state $v \in V$ naturally define a finite $V$-tree $\mathcal{T}_{\sigma, v}$ with root $v$ : given a state $s$, a word $t=u \cdot s$ is in $\mathcal{T}_{\sigma, v}$ iff $u \in \mathcal{T}_{\sigma, v}$ and, seeing $u$ as a finite run, we have either last $(u)=v^{\prime} \in V_{\diamond}$ and $\left(v^{\prime}, s\right) \in \sigma(u)$, or last $(u)=v^{\prime} \in V_{\square}$ and $\left(v^{\prime}, s\right) \in E$. In this tree, the height of the root coincides with the length of a longest run generated by the multi-strategy $\sigma$ from $v$. Since the multi-strategy $\sigma$ is winning from $v$, all branches are finite, and all leaves of $\mathcal{T}_{\sigma, v}$ are occurrences of $\odot$. The union of all trees $\mathcal{T}_{\sigma, v}$ (for $v$ a winning state) defines a forest $\mathcal{T}_{\sigma}$.

Conversely, every $V$-forest $\mathcal{T}=(T, W)$ with $W \subseteq V$ satisfying the following conditions naturally defines a winning multi-strategy $\sigma_{\mathcal{T}}$ (viewing each node $t \in T$ as a run of $\mathrm{G})$ :

- if last $(t)=v^{\prime} \in V_{\square}, t \cdot s \in T$ iff $\left(v^{\prime}, s\right) \in E$;

- if last $(t)=v^{\prime} \in V_{\diamond}$ and $t \cdot s \in T$, then $\left(v^{\prime}, s\right) \in E$. In that case we set $\sigma_{\mathcal{T}}(t)=\left\{\left(v^{\prime}, s\right) \in E \mid t \cdot s \in T\right\}$

- if $t$ is maximal, then last $(t)=\odot$.

Lemma 5. Assume that we are given an optimal winning multi-strategy $\sigma$. We can effectively construct in polynomial time a memoryless multi-strategy $\sigma^{\prime}$, which is winning and optimal.

\footnotetext{
${ }^{5}$ An appropriate measure will be assigned to every node of the forest.
} 
Proof. Assume that $W$ is the set of winning states. Let $\mathcal{T}$ be the forest representing the multi-strategy $\sigma$ (its set of roots is $W$ ). Since $\sigma$ is winning from every state in $W$, all branches of the forest are finite. For every node $t$ of $\mathcal{T}$, we define $\gamma_{\mathcal{T}}(t)$ as the residual penalty of $\sigma$ after prefix $t$. Formally, $\gamma_{\mathcal{T}}(t)=$ penalty $(\sigma, t)$. Obviously, for all $v \in V$, we have penalty $(\sigma, v)=\gamma_{\mathcal{T}}(v)$.

We will consider a measure $\mu_{\mathcal{T}}$ on the set of nodes of the forest $\mathcal{T}$ as follows: if $t$ is a node of $\mathcal{T}$, we let $\mu_{\mathcal{T}}(t)=\left(\gamma_{\mathcal{T}}(t)\right.$, height $\left.t_{\mathcal{T}}(t)\right)$.

We say that no memory is required for state $v$ in $\mathcal{T}$ if, for every two nodes $t$ and $t^{\prime}$ that are occurrences of $v$, the subtree of $\mathcal{T}$ rooted at $t$ and the subtree of $\mathcal{T}$ rooted at $t^{\prime}$ are identical. Note that in that case, $\mu_{\mathcal{T}}(t)=\mu_{\mathcal{T}}\left(t^{\prime}\right)$.

For every $0 \leq i \leq|W|$, we inductively build in polynomial time a forest $\mathcal{T}^{i}$ and a set $M_{i} \subseteq W$ containing $i$ elements, such that:

(a) $\mathcal{T}^{i}$ represents an optimal winning multi-strategy from all the states of $W$;

(b) for every $v \in M_{i}$, no memory is required for $v$ in $\mathcal{T}^{i}$, and for every node $t^{\prime}$ which is a descendant of some node that is an occurrence of $v$, letting $v^{\prime}=$ last $\left(t^{\prime}\right)$, it holds $v^{\prime} \in M_{i}$.

Intuitively, each $\mathcal{T}^{i}$ will be the forest of a winning optimal multi-strategy $\sigma_{i}$, and each $M_{i}$ will be a set of states from which $\sigma_{i}$ is memoryless (i.e., $\sigma_{i}$ is memoryless from the states in $M_{i}$, and from the states that occur in the outcomes from these states). In the end, the forest $\mathcal{T}^{|W|}$ represents a multi-strategy $\sigma^{\prime}$ which is memoryless, optimal and winning from every state of the game.

\section{Discounted and Mean Penalty Games}

\subsection{Discounted and mean penalties of multi-strategies}

We have proposed a way to measure the permissivity of winning strategies in games, by summing penalties for blocking edges in the graph. It can be interesting to consider that blocking an edge early in a run is more restrictive than blocking an edge later. A classical way to represent this is to consider a discounted version of the penalty of a multi-strategy, which we now define.

Discounted penalties of multi-strategies. Let $\mathrm{G}=\left(V_{\square}, V_{\diamond}, E\right.$, weight $)$ be a weighted game, $\sigma$ be a winning (w.r.t. the reachability objective) multi-strategy, and $\varrho_{0}$ be a finite outcome of $\sigma$. Given a discount factor $\lambda \in(0,1)$, the discounted penalty of $\sigma$ after $\varrho_{0}$ (w.r.t. $\lambda$ ), denoted penalty ${ }^{\lambda}\left(\sigma, \varrho_{0}\right)$, is defined as $\sup \left\{\right.$ penalty ${ }_{\sigma, \varrho_{0}}^{\lambda}(\varrho) \mid \varrho \in$ Out $\left._{\mathrm{G}}^{\max }\left(\sigma, \varrho_{0}\right)\right\}$, where penalty ${ }_{\sigma, \varrho_{0}}^{\lambda}(\varrho)$ is inductively defined for all $\varrho \in \operatorname{Out}_{\mathrm{G}}\left(\sigma, \varrho_{0}\right)$ as follows:

- penalty ${ }_{\sigma, \varrho_{0}}^{\lambda}\left(\varrho_{0}\right)=0$;

- if last $(\varrho) \notin V_{\diamond}$ and $(\operatorname{last}(\varrho), v) \in E$, then penalty ${ }_{\sigma, \varrho_{0}}^{\lambda}(\varrho \rightarrow v)=$ penalty $_{\sigma, \varrho_{0}}^{\lambda}(\varrho)$;

- if last $(\varrho) \in V_{\diamond}$ and $($ last $(\varrho), v) \in \sigma(\varrho)$, then penalty ${ }_{\sigma, \varrho_{0}}^{\lambda}(\varrho \rightarrow v)$ is defined as

$$
\operatorname{penalty}_{\sigma, \varrho_{0}}^{\lambda}(\varrho)+\lambda^{|\varrho|-\left|\varrho_{0}\right|} \cdot \sum_{\left(\operatorname{last}(\varrho), v^{\prime}\right) \in(E \backslash \sigma(\varrho))} \operatorname{weight}\left(\operatorname{last}(\varrho), v^{\prime}\right) .
$$


We also define the discounted penalty along infinite runs, as being the limit (which necessarily exists as $\lambda<1$ ) of the penalties along the finite prefixes.

We write opt_penalty ${ }^{\lambda}\left(\varrho_{0}\right)$ for the optimal discounted penalty (w.r.t. $\lambda$ ) Player $\diamond$ can ensure after $\varrho_{0}$ while reaching $\odot$ :

$$
\text { opt_penalty }^{\lambda}\left(\varrho_{0}\right)=\inf \left\{\text { penalty }^{\lambda}\left(\sigma, \varrho_{0}\right) \mid \sigma \text { winning multi-strategy after } \varrho_{0}\right\} \text {. }
$$

Given $\varepsilon \geq 0$ and $\lambda \in(0,1)$, a multi-strategy $\sigma$ is said $\varepsilon$-optimal for discount factor $\lambda$ after $\varrho_{0}$ if it is winning after $\varrho_{0}$ and

$$
\text { penalty }^{\lambda}\left(\sigma, \varrho_{0}\right) \leq \text { opt_penalty }^{\lambda}\left(\varrho_{0}\right)+\varepsilon \text {. }
$$

Again, optimality is a shorthand for 0-optimality. Finally, a multi-strategy is $\varepsilon$-optimal for discount factor $\lambda$ if it is $\varepsilon$-optimal for $\lambda$ from any winning state.

Discounted cost in weighted games. As in Section 2.1, we recall the usual notion $\operatorname{cost}^{\lambda}$ of discounted cost of runs in a weighted game $[\mathrm{ZP} 96]^{6}$ :

$-\operatorname{cost}^{\lambda}(v)=0$

$-\operatorname{cost}^{\lambda}(\varrho \stackrel{e}{\rightarrow})=\operatorname{cost}^{\lambda}(\varrho)+\lambda^{|\varrho|-1} \cdot \operatorname{weight}(e) ;$

Then we define $\operatorname{cost}^{\lambda}\left(\sigma, \varrho_{0}\right)=\sup \left\{\operatorname{cost}^{\lambda}(\varrho) \mid \varrho \in \operatorname{Out}_{\mathrm{G}}^{\max }\left(\sigma, \varrho_{0}\right)\right\}$. Those games are symmetric, and later we will sometimes take the point-of-view of Player $\square$ whose objective will be to maximize the discounted cost: given a strategy $\sigma$ for Player $\square$, we then define $\operatorname{cost}^{\lambda}\left(\sigma, \varrho_{0}\right)=\inf \left\{\operatorname{cost}^{\lambda}(\varrho) \mid \varrho \in \operatorname{Out}_{\mathrm{G}}^{\max }\left(\sigma, \varrho_{0}\right)\right\}$.

Computation and decision problems. As in the previous section, our aim is to compute (almost-)optimal multi-strategies. The optimal reachability problem with discounted penalty is the following: given a weighted game G, a rational number $c$, a discount factor $\lambda \in(0,1)$, and a state $v \in V$, does there exist a multi-strategy $\sigma$ for Player $\diamond$ such that penalty ${ }^{\lambda}(\sigma, v) \leq c$. The transformations between penalties and costs depicted in Section 2.2 are still possible in the discounted setting. The only point is that in both cases, each single transition gives rise to two consecutive transitions, so that we must consider $\sqrt{\lambda}$ as the new discounting factor ${ }^{7}$.

\subsection{Some examples}

As far as the existence of an optimal multi-strategy is concerned, the discounted case is more challenging as the results of the previous section do not hold. In particular, we exemplify on Figures 4 and 5 the fact that optimal multistrategies do not always exist, and when they exist, they cannot always be made memoryless.

Lemma 6. There exists weighted games for which there is no optimal winning multi-strategy under discounted penalties.

\footnotetext{
${ }^{6}$ Note that we have dropped the normalization factor $(1-\lambda)$, which is only important to relate $\lambda$-discounted values to mean values (by making $\lambda$ tend to 1 ) [ZP96].

${ }^{7}$ For the reduction of Lemma 3, the penalty is also multiplied by $\sqrt{\lambda}$
} 


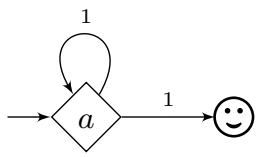

Fig. 4. No optimal discounted multi-strategy

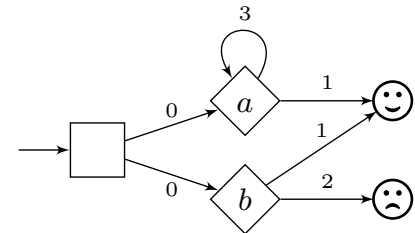

Fig. 5. No memoryless optimal discounted multi-strategy

Lemma 7. There are weighted games under discounted penalties for which there exist a memoryful optimal winning multi-strategy but no memoryless one.

\subsection{A pair of memoryless strategies is sufficient}

We prove here that there always exist $\varepsilon$-optimal multi-strategies that are made of two memoryless multi-strategies. Roughly, the first multi-strategy aims at lengthening the path (so that the coefficient $\lambda^{|\varrho|}$ will be small) without increasing the penalty, and the second multi-strategy aims at reaching the final state.

To this aim, we need to first study the multi-strategy problem in the setting where there is no reachability objective. Let $\mathrm{G}$ be a finite weighted game, $\lambda \in(0,1)$, and $c \in \mathbb{Q}$. The optimal discounted-penalty problem consists in deciding whether there is a multi-strategy for Player $\diamond$ for which the $\lambda$-discounted penalty along any maximal (finite or infinite) outcome is less than or equal to $c$.

Theorem 8. The optimal discounted-penalty problem is in NP $\cap$ coNP, and is PTIME-hard.

The proof of this theorem relies on known results in classical discounted games [ZP96,Jur98], uses the transformation of Lemma 2 and monotonicity properties already used in the proof given in section 3.1.

Proof. We let $\mathrm{G}=\left(V_{\square}, V_{\diamond}, E\right.$, weight $)$ be a finite weighted game with no incoming transitions to $\odot$, and let $c \in \mathbb{Q}$. Applying the transformation of Lemma 2 to the discounted case, we get an exponential-size weighted game $\mathrm{G}^{\prime}=\left(V_{\square}^{\prime}, V_{\diamond}^{\prime}\right.$, $E^{\prime}$, weight ${ }^{\prime}$ ) with $V_{\square} \subseteq V_{\square}^{\prime}$ and $V_{\diamond}=V_{\diamond}^{\prime}$ such that for every $v \in V_{\square} \cup V_{\diamond}$, Player $\diamond$ has a winning multi-strategy from $v$ in $\mathrm{G}$ with discounted penalty no more than $c$ (for discount $\lambda$ ) iff Player $\diamond$ has a winning strategy from $v$ in $\mathrm{G}^{\prime}$ with discounted cost no more than $c$ (for discount $\sqrt{\lambda}$ ).

From [ZP96], Player $\diamond$ has a memoryless optimal strategy in $\mathrm{G}^{\prime}$. The NP algorithm is then as follows: guess such a memoryless strategy $\sigma \diamond$ for Player $\diamond$, i.e. for every $v \in V_{\diamond}$ guess a subset $F \subseteq v E$ and set $\sigma_{\diamond}(v)=(v, F)$. Removing from $G^{\prime}$ transitions that have not been chosen by $\sigma \diamond$ yields a polynomial-size graph $G^{\prime \prime}$, in which we can compute in polynomial time the maximal discounted cost, which corresponds to $\operatorname{cost}^{\sqrt{\lambda}}\left(\sigma_{\diamond}, v\right)$. The graph $\mathrm{G}^{\prime \prime}$ can be computed from $\mathrm{G}$ without explicitly building $G^{\prime}$, so that our procedure runs in polynomial time.

Membership in coNP is harder, and we only give a sketch of proof here. The game $G^{\prime}$ is memoryless determined [ZP96], which means that for every 
$c \in \mathbb{Q}$, for every state $v \in V_{\square}^{\prime} \cup V_{\diamond}^{\prime}$, either Player $\diamond$ has a memoryless strategy $\sigma_{\diamond}$ with $\operatorname{cost}^{\sqrt{\lambda}}\left(\sigma_{\diamond}, v\right) \leq c$, or Player $\square$ has a memoryless strategy $\sigma_{\square}$ with $\operatorname{cost}^{\sqrt{\lambda}}\left(\sigma_{\square}, v\right)>c$. Our coNP algorithm consists in guessing a memoryless strategy for Player $\square$ that achieves cost larger than $c$. However, Player $\square$ controls exponentially many states in $\mathrm{G}^{\prime}$, so that we will guess a succinct encoding of her strategy, based on the following observation: there is a (preference) order on the states in $V_{\square} \cup V_{\diamond}^{8}$ so that, in states of the form $(v, F)$, the optimal strategy for Player $\square$ consists in playing the "preferred" state of $F$ (w.r.t. the order). In other words, the strategy in those states can be defined in terms of an order on the states, which can be guessed in polynomial time.

Once such a strategy has been chosen non-deterministically, it then suffices to build a polynomial-size graph $\mathrm{G}^{\prime \prime}$ in which the cost of the strategy $\sigma_{\square}$ corresponds to the minimal discounted cost of Player $\square$ in $\mathrm{G}^{\prime}$.

Hardness in PTIME directly follows from Lemma 3.

Remark 1. This problem could be extended with safety condition: the aim is then to minimize the discounted penalty while avoiding some bad states. An easy adaptation of the previous proof yields the very same results for this problem.

Definition 9. Let $\sigma_{1}$ and $\sigma_{2}$ be two memoryless multi-strategies, and $k \in \mathbb{N}$. The multi-strategy $\sigma=\sigma_{1}^{k} \cdot \sigma_{2}^{*}$ is defined, for each $\varrho$ such that last $(\varrho) \in V_{\diamond}$, as:

- if $|\varrho|<k$, then $\sigma(\varrho)=\sigma_{1}(\varrho)$;

- if $|\varrho| \geq k$, then $\sigma(\varrho)=\sigma_{2}(\varrho)$.

Theorem 10. Let $\mathrm{G}=\left(V_{\square}, V_{\diamond}, E\right.$, weight $)$ be a finite weighted game with a reachability objective, and $\lambda \in(0,1)$. Then there exist two memoryless multistrategies $\sigma_{1}$ and $\sigma_{2}$ such that, for any $\varepsilon>0$, there is an integer $k$ such that the multi-strategy $\sigma_{1}^{k^{\prime}} \cdot \sigma_{2}^{*}$ is $\varepsilon$-optimal (w.r.t. $\lambda$-discounted penalties) for any $k^{\prime} \geq k$.

Proof. This is proved together with the following lemma:

Lemma 11. Let $\mathrm{G}=\left(V_{\square}, V_{\diamond}, E\right.$, weight $)$ be a finite weighted game with a reachability objective, $\lambda \in(0,1)$, and $c \in \mathbb{Q}$. Then $(\mathrm{G}, \lambda, c)$ is a positive instance of the optimal discounted-penalty problem iff for any $\varepsilon>0,(\mathrm{G}, \lambda, c+\varepsilon)$ is a positive instance of the optimal reachability problem with discounted penalty

Proof. From the remark following the proof of Theorem 8, there is a memoryless optimal multi-strategy $\sigma_{1}$ all of whose maximal outcomes have $\lambda$-discounted penalty less than or equal to $c$, and never visit losing states. Let $\sigma_{2}$ be a memoryless winning multi-strategy for the reachability objective, and let $c_{2}$ be the maximal penalty accumulated along an outcome of $\sigma_{2}$. Let $\varepsilon>0$, and $k \in \mathbb{N}$ such that $\lambda^{k} \cdot c_{2} \leq \varepsilon$. Then for any $k^{\prime}>k$, the multi-strategy $\sigma_{1}^{k^{\prime}} \cdot \sigma_{2}^{*}$ is winning, and the $\lambda$-discounted penalty of any outcome is at most $c+\lambda^{k^{\prime}} \cdot c_{2} \leq c+\varepsilon$.

${ }^{8}$ Which will be given by ordering the values given by the classical optimality equations [Jur98] in $\mathrm{G}^{\prime}$. 
Conversely, let $\varepsilon>0$, and $\sigma$ be a winning multi-strategy achieving discounted penalty no more than $c+\varepsilon$. Then in particular, $\sigma$ achieves discounted penalty less than or equal to $c+\varepsilon$ along all of its outcomes, so that $(G, \lambda, c+\varepsilon)$ is a positive instance of the optimal discounted-penalty problem (for any $\varepsilon>0$ ). From Theorem 8, this problem admits a (truly) optimal memoryless multi-strategy, so that there must exist a multi-strategy achieving discounted penalty less than or equal to $c$ along all of its outcomes.

Theorem 12. The optimal reachability problem with discounted penalty is in $\mathrm{NP} \cap$ coNP, and is PTIME-hard.

Remark 2. It can be observed that the results of this section extend to discountedcost games with reachability objectives (without the exponential gap due to the first transformation of weighted games with penalties). In particular, those games admit almost-optimal strategies made of two memoryless strategies, and the corresponding decision problem is equivalent to classical discounted-payoff games.

\subsection{Extension to the mean penalty of multi-strategies}

We also define the mean penalty of a multi-strategy $\sigma$ from state $v$, denoted mean_penalty $(\sigma, v)$, as $\sup \left\{\right.$ mean_penalty $_{\sigma}(\varrho) \mid \varrho \in \operatorname{Out}_{\mathrm{G}}(\sigma, v), \varrho$ maximal $\}$, where

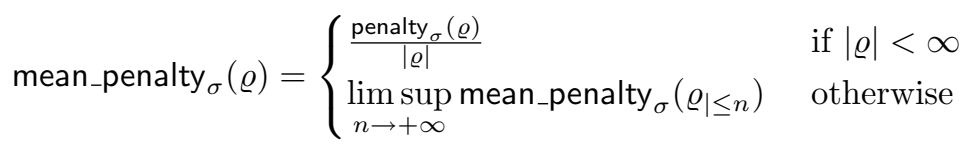

where $\varrho_{\mid \leq n}$ is the prefix of length $n$ of $\varrho$. The notion of $\varepsilon$-optimality, for $\varepsilon \geq 0$, is defined as previously. Using the same lines of arguments as earlier, we get:

Theorem 13. Let $\mathrm{G}=\left(V_{\square}, V_{\diamond}, E\right.$, weight) be a finite weighted game with reachability objectives, in which all states in $V_{\square} \cup V_{\diamond}$ are winning. There exist two memoryless multi-strategies $\sigma_{1}$ and $\sigma_{2}$ such that, for any $\varepsilon>0$, there exists $k$ so that the multi-strategy $\sigma_{1}^{k^{\prime}} \cdot \sigma_{2}^{*}$ is $\varepsilon$-optimal (w.r.t. mean penalties) for any $k^{\prime} \geq k$.

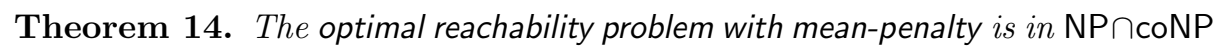
and is PTIME-hard.

Remark 3. Again, this result extends to mean-cost games with reachability objectives, which thus admit almost-optimal strategies made of two memoryless strategies. Surprisingly, the same phenomenon has been shown to occur in meanpayoff parity games [CHJ05], but the corresponding strategy can be made fully optimal thanks to the infiniteness of the outcomes.

\section{Conclusion and future work}

We have proposed an original quantitative approach to the permissivity of (multi-)strategies in two-player games with reachability objectives, through a natural notion of penalty given to the player for blocking edges. We have proven that 
most permissive strategies exist and can be chosen memoryless in the case where penalties are added up along the outcomes, and proposed a PTIME algorithm for computing such an optimal strategy. When considering discounted sum or mean penalty, we have proved that we must settle for almost-optimal strategies, which are built from two memoryless strategies. The resulting algorithm is in NP $\cap$ coNP. This is rather surprising as the natural way of encoding multi-strategies in classical weighted games entails an exponential blowup.

Besides the naturalness of multi-strategies, our initial motivation underlying this work (and the aim of our future works) is in the domain of timed games [AMPS98,BCD $\left.{ }^{+} 07\right]$ : in that setting, strategies are often defined as functions from executions to pairs $(t, a)$ where $t$ is a real number and $a$ an action. This way of defining strategies goes against the paradigm of implementability [DDR04], as it requires infinite precision. We plan to extend the work reported here to the timed setting, where penalties would depend on the precision needed to apply the strategy. Also, as stated in [CHJ05], we believe that games with mixed objectives are interesting on their own, which gives another direction of research for future work. This catches up with related works on quantitative extensions of ATL.

\section{References}

[AMPS98] E. Asarin, O. Maler, A. Pnueli, and J. Sifakis. Controller synthesis for timed automata. In Proc. IFAC Symposium on System Structure and Control, p. 469-474. Elsevier Science, 1998.

$\left[\mathrm{BCD}^{+}\right.$07] G. Behrmann, A. Cougnard, A. David, E. Fleury, K. G. Larsen, and D. Lime. UPPAAL-Tiga: Time for playing games! In Proc. 19th Intl Conf. on Computer Aided Verification (CAV'O'7), LNCS 4590, p. 121-125. Springer, 2007.

[BJW02] J. Bernet, D. Janin, and I. Walukiewicz. Permissive strategies: From parity games to safety games. Inf. Théor. et Applications, 36(3):261-275, 2002.

[CHJ05] K. Chatterjee, Th. A. Henzinger, and M. Jurdziński. Mean-payoff parity games. In Proc. 20th Annual Symposium on Logic in Computer Science (LICS'05). IEEE Computer Society Press, 2005.

[DDR04] M. De Wulf, L. Doyen, and J.-F. Raskin. Almost ASAP semantics: From timed models to timed implementations. In Proc. 7th Intl Workshop on Hybrid Systems: Computation and Control (HSCC'04), LNCS 2993, p. 296310. Springer, 2004.

[HP06] T. A. Henzinger and V. S. Prabhu. Timed alternating-time temporal logic. In Proc. 4th Intl Conf. on Formal Modeling and Analysis of Timed Systems (FORMATS'06), LNCS 4202, p. 1-17. Springer, 2006.

[Jur98] M. Jurdziński. Deciding the winner in parity games is in UP $\cap$ coUP. Information Processing Letters, 68(3):119-124, 1998.

[LMO06] F. Laroussinie, N. Markey, and G. Oreiby. Model checking timed ATL for durational concurrent game structures. In Proc. 4th Intl Conf. on Formal Modeling and Analysis of Timed Systems (FORMATS'06), LNCS 4202, p. 245-259. Springer, 2006.

[Lut08] M. Luttenberger. Strategy iteration using non-deterministic strategies for solving parity games. Research Report cs.GT/0806.2923, arXiv, 2008.

[PR05] S. Pinchinat and S. Riedweg. You can always compute maximally permissive controllers under partial observation when they exist. In Proc. 24th American Control Conf. (ACC'05), p. 2287-2292, 2005. 
[Tho02] W. Thomas. Infinite games and verification. In Proc. 14th Intl Conf. on Computer Aided Verification (CAV'02), LNCS 2404, p. 58-64. Springer, 2002.

[ZP96] U. Zwick and M. Paterson. The complexity of mean payoff games on graphs. Theoretical Computer Science, 158(1-2):343-359, 1996.

\section{A Proof of Lemmas 2 and 3}

Lemma 2. For every weighted game $\mathrm{G}=\left(V_{\square}, V_{\diamond}, E\right.$, weight $)$, we can construct an exponential-size weighted game $\mathrm{G}^{\prime}=\left(V_{\square}^{\prime}, V_{\diamond}^{\prime}, E^{\prime}\right.$, weight $\left.{ }^{\prime}\right)$ such that $V_{\square} \subseteq V_{\square}^{\prime}$, $V_{\diamond} \subseteq V_{\diamond}^{\prime}$ and, for any state $v \in V_{\square} \cup V_{\diamond}$ and any bound $c$, Player $\diamond$ has a winning multi-strategy with penalty $c$ from $v$ in $\mathrm{G}$ iff she has a winning strategy with cost $c$ from $v$ in $\mathrm{G}^{\prime}$.

Proof. The weighted game $\mathrm{G}^{\prime}=\left(V_{\square}^{\prime}, V_{\diamond}^{\prime}, E^{\prime}\right.$, weight $\left.{ }^{\prime}\right)$ is defined as follows (see Fig. 2 for an example):

- the set of states of Player $\diamond$ is unchanged, while the set of states of Player $\square$ is augmented with $\left\{(v, F) \mid v \in V_{\diamond}, F \subseteq v E\right\}$;

- $E^{\prime}$ is the (disjoint) union of three kinds of transitions:

(1) transitions in $E \cap\left(V_{\square} \times V\right)$,

(2) transitions of the form $\left((v, F), v^{\prime}\right)$ for each $\left(v, v^{\prime}\right) \in F$,

(3) transitions of the form $(v,(v, F))$ for each $v \in V_{\diamond}$ and $F \subseteq v E$;

- the weight function weight ${ }^{\prime}$ is defined as follows:

- each edge $e$ of type (1) has weight weight $(e)$;

- transitions of type (2) have weight 0 ;

- each transition $(v,(v, F))$ of type (3) has weight $\sum_{e \in v E \backslash F}$ weight $(e)$.

We now prove the correctness of this construction. Let $\sigma$ be a winning multistrategy for Player $\diamond$ in G. Given a run $\varrho^{\prime}$ in $\mathrm{G}^{\prime}$ such that last $\left(\varrho^{\prime}\right) \in V_{\diamond}^{\prime}$, we consider the run $\varrho$ obtained from $\varrho^{\prime}$ by removing every second state. This is a finite run of G, ending in $V_{\diamond}$. We let $\sigma^{\prime}\left(\varrho^{\prime}\right)=\left(\operatorname{last}\left(\varrho^{\prime}\right), \sigma(\varrho)\right)$. By construction, the cost of this transition corresponds to the penalty of playing $\sigma$ from $\varrho$. Then Player $\square$ in $\mathrm{G}^{\prime}$ can choose one of the edges in $\sigma(\varrho)$, and switch to the corresponding state. In the end, any outcome $\varrho^{\prime}$ of $\sigma^{\prime}$ in $\mathrm{G}^{\prime}$ with cost $d$ corresponds to an outcome of $\sigma$ in $\mathrm{G}$ with penalty $d$, and conversely. This entails that $\sigma^{\prime}$ is winning and has cost the penalty of $\sigma$.

For the other direction, we define $\sigma$ inductively (on the length of the history) from $\sigma^{\prime}$, enforcing that the outcomes of $\sigma$ in $\mathrm{G}$ of length $n$ correspond (one-toone) to the outcomes of $\sigma^{\prime}$ in $\mathrm{G}^{\prime}$ of length $2 n-1$, and that this correspondence preserves the quantity (cost vs. penalty) of the outcomes:

- for $v \in V_{\diamond}$, letting $\sigma^{\prime}(v)=(v, F)$, we define $\sigma(v)=F$ (which is a set of edges leaving $v$ ). By construction, the penalty assigned to $\sigma$ in $v$ is the weight of the transition $(v, F)$. The correspondence between outcomes is thus satisfied;

- given an outcome $\varrho$ of $\sigma$ of length $n$, we define $\sigma(\varrho)$ as the set of edges indicated by $\sigma^{\prime}\left(\varrho^{\prime}\right)$, where $\varrho^{\prime}$ is the outcome of $\sigma^{\prime}$ corresponding to $\varrho$. Again, the correspondence between outcomes holds with this definition. 
The definition of $\sigma$ on histories that are not outcomes of $\sigma$ is irrelevant and can be set arbitrarily. Thanks to the correspondence between outcomes, $\sigma$ is winning (assuming that $\sigma^{\prime}$ is) and its penalty is the cost of $\sigma^{\prime}$.

Lemma 3. For every weighted game $\mathrm{G}^{\prime}=\left(V_{\square}^{\prime}, V_{\diamond}^{\prime}, E^{\prime}\right.$, weight $\left.{ }^{\prime}\right)$, we can construct a polynomial-size weighted game $\mathrm{G}=\left(V_{\square}, V_{\diamond}, E\right.$, weight $)$ such that $V_{\square}^{\prime} \subseteq V_{\square}$, $V_{\diamond}^{\prime} \subseteq V_{\diamond}$, and for any state $v \in V_{\square}^{\prime} \cup V_{\diamond}^{\prime}$ and any value $c$, Player $\diamond$ has a winning strategy with cost $c$ from $v$ in $\mathrm{G}^{\prime}$ iff she has a winning multi-strategy with penalty $c$ from $v$ in $\mathrm{G}$.

Proof. The penalty game $\mathrm{G}=\left(V_{\square}, V_{\diamond}, E\right.$, weight $)$ is defined as follows:

- the set of states of Player $\square$ is unchanged, while $V_{\diamond}=V_{\diamond}^{\prime} \cup V_{E^{\prime}}$ where $V_{E^{\prime}}=\left\{v_{e} \mid e \in E^{\prime}\right\}$ is a set disjoint from $V_{\diamond}^{\prime}$. In other words we add one state for each edge of $G^{\prime}$;

$-E$ is the (disjoint) union of three kinds of edges:

(1) edges of the form $\left(v, v_{e}\right)$ for each $v \in V$ and each $e \in v E^{\prime}$,

(2) edges of the form $\left(v_{e}, v\right)$ for each $v \in V$ and each $e \in E^{\prime} v$,

(3) edges of the form $\left(v_{e}, \odot\right)$ for each $e \in E^{\prime}$;

- the weight function weight is defined as follows:

- each edge $\left(v, v_{e}\right)$ or $\left(v_{e}, v\right)$ of type (1) or (2) has weight 0 ,

- each edge $\left(v_{e}, \odot\right)$ of type (3) has weight weight ${ }^{\prime}(e)$.

Now, a winning strategy $\sigma^{\prime}$ for Player $\diamond$ in the original game $\mathrm{G}^{\prime}$ can be transformed into a winning multi-strategy $\sigma$ (which is actually a deterministic strategy) in $\mathrm{G}$ such that $\operatorname{cost}\left(\sigma^{\prime}, v\right)=$ penalty $(\sigma, v)$ for all $v \in V_{\square}^{\prime} \cup V_{\diamond}^{\prime}$. The transformation is defined inductively (on the length of the history) as follows:

- if $v \in V_{\diamond}^{\prime}$, the state corresponds to a state in $\mathrm{G}^{\prime}$ and we set $\sigma(v)=\left(v, v_{e}\right)$ where $e=\sigma^{\prime}(v)$;

- if $v=v_{e} \in V_{E^{\prime}}$ and $e=\left(v^{\prime}, v^{\prime \prime}\right)$ then $\sigma(v)=\left(v_{e}, v^{\prime \prime}\right)$;

- if $\varrho \in \operatorname{Out}_{\mathrm{G}}(\sigma, v)$ with last $(\varrho)=v_{e} \in V_{E^{\prime}}$ and $e=\left(v^{\prime}, v^{\prime \prime}\right)$ then $\sigma(\varrho)=\left(v_{e}, v^{\prime \prime}\right)$;

- if $\varrho \in \operatorname{Out}_{\mathrm{G}}(\sigma, v)$ with last $(\varrho)=v^{\prime} \in V_{\diamond}^{\prime}$, then by removing every second state from $\varrho$ we have a run $\varrho^{\prime} \in \operatorname{Out}_{G^{\prime}}\left(\sigma^{\prime}, v\right)$, and we can thus define $\sigma(\varrho)=\left(v^{\prime}, v_{e}\right)$ with $e=\sigma^{\prime}\left(\varrho^{\prime}\right)$. Furthermore, it is easy to see that the cost of $\varrho^{\prime}$ is precisely the penalty of $\varrho$ in $\mathrm{G}$;

- if $\forall v, \varrho \notin \operatorname{Out}_{\mathrm{G}}(\sigma, v)$ then $\sigma(\varrho)$ doesn't matter (we can take $\sigma(\varrho)=\varnothing$ ).

The strategy $\sigma^{\prime}$ is winning in $\mathrm{G}^{\prime}$ iff $\sigma$ is winning in $\mathrm{G}$, and the cost/penalty of both strategies coincide.

Conversely, a winning multi-strategy $\sigma$ for Player $\diamond$ in G can be transformed into a winning strategy $\sigma^{\prime}$ in $\mathrm{G}^{\prime}$ such that $\operatorname{cost}\left(\sigma^{\prime}, v\right)=$ penalty $(\sigma, v)$ for all $v \in$ $V_{\square}^{\prime} \cup V_{\diamond}^{\prime}$. 
Since we want to build a strategy from a multi-strategy we first need to fix the non-deterministic choices allowed in $\sigma$. We thus define a deterministic ${ }^{9}$ multistrategy $\sigma_{1}$ in $\mathrm{G}$ such that for every $v \in V_{\square} \cup V_{\diamond}$, penalty $\left(\sigma_{1}, v\right)=$ penalty $(\sigma, v)$. By definition of penalties, and given that $\sigma$ is winning, for every $v \in V_{\square}^{\prime} \cup V_{\diamond}^{\prime}$ the penalty penalty $(\sigma, v)$ is the maximum over all (finite) paths in $\operatorname{Out}_{\mathrm{G}}^{\max }(\sigma, v)$ of the penalty along a path. Let $\varrho_{v}$ be a path achieving such a maximum for $v$. We now give the multi-strategy $\sigma_{1}$ :

- if $\varrho \in \operatorname{Out}_{G}(\sigma, v)$ with last $(\varrho)=v^{\prime} \in V_{E^{\prime}}$ then $\sigma(\varrho)$ contains just one edge (the one not leading to the bad state) and is 'deterministic'. In that case we set $\sigma_{1}(\varrho)=\sigma(\varrho)$;

- if $\varrho \in \operatorname{Out}_{\mathrm{G}}(\sigma, v)$ with last $(\varrho)=v^{\prime} \in V_{\diamond}^{\prime}$ and $\varrho$ is a prefix of $\varrho_{v}\left(i . e . \varrho_{v}=\varrho v_{e} \varrho^{\prime}\right)$, we choose for $\sigma_{1}(\varrho)$ the following edge along $\varrho_{v}$, i.e. $\sigma_{1}(\varrho)=\left\{\left(v^{\prime}, v_{e}\right)\right\}$;

- if $\varrho \in \operatorname{Out}_{\mathrm{G}}(\sigma, v)$ with last $(\varrho)=v^{\prime} \in V_{\diamond}^{\prime}$ and $\varrho$ is not a prefix of $\varrho_{v}$, then we choose arbitrarily an edge $e$ in $\sigma(\varrho)$ and set $\sigma_{1}(\varrho)=e$;

- if for every $v \in V, \varrho \notin \operatorname{Out}_{\mathrm{G}}(\sigma, v)$, since $\operatorname{Out}_{\mathrm{G}}\left(\sigma_{1}, v\right) \subseteq \operatorname{Out}_{\mathrm{G}}(\sigma, v)$ the definition of $\sigma_{1}(\varrho)$ doesn't matter and we set $\sigma_{1}(\varrho)=\varnothing$.

We clearly have a deterministic multi-strategy, but need to prove that the penalties have been preserved. First Out ${ }_{\mathrm{G}}^{\max }\left(\sigma_{1}, v\right) \subseteq \operatorname{Out}_{\mathrm{G}}^{\max }(\sigma, v)$, and since we have only removed edges of weight 0 , the penalties of the remaining runs have not changed. Taking the maximum of penalties over a smaller set we get penalty $\left(\sigma_{1}, v\right) \leq$ penalty $(\sigma, v)$. On the other hand the path $\varrho_{v}$ that achieved the maximal penalty is in $\operatorname{Out}_{\mathrm{G}}^{\max }\left(\sigma_{1}, v\right)$. Since its penalty has been preserved, we have penalty $\left(\sigma_{1}, v\right) \geq$ penalty $(\sigma, v)$ and thus the equality.

The last step is to define a winning strategy $\sigma^{\prime}$ in $\mathrm{G}^{\prime}$ based on $\sigma_{1}$ and preserving the cost/penalty. By construction, for every edge $e=\left(v, v^{\prime}\right) \in E^{\prime}$ there exist two edges $\left(v, v_{e}\right)$ and $\left(v_{e}, v^{\prime}\right)$ in $E$, thus we can associate to every run $\varrho^{\prime}=v_{0} v_{1} \ldots v_{k}$ in Runs $\mathrm{G}^{\prime}$ a unique run $\iota\left(\varrho^{\prime}\right)=v_{0} v_{e_{0}} v_{1} v_{e_{1}} \ldots v_{e_{k-1}} v_{k}$ in Runs $\operatorname{Ru}_{\mathrm{G}}$ with $e_{i}=\left(v_{i}, v_{i+1}\right)$ for every $0 \leq i<k$. We now inductively define the strategy $\sigma$ :

- if $v \in V_{\diamond}^{\prime}$, we set $\sigma^{\prime}(v)=e$ where $\sigma_{1}(v)=\left\{\left(v, v_{e}\right)\right\}$;

- if $\varrho^{\prime} \in \operatorname{Out}_{\mathrm{G}^{\prime}}\left(\sigma^{\prime}, v\right)$ with last $\left(\varrho^{\prime}\right)=v^{\prime} \in V_{\diamond}^{\prime}$ we set $\sigma^{\prime}\left(\varrho^{\prime}\right)=e$, where $\sigma_{1}(\iota(\varrho))=$ $\left\{\left(v^{\prime}, v_{e}\right)\right\}$.

By construction of G, from a state $v$ in $V_{\diamond}^{\prime} \subseteq V_{\diamond}$, the choice of an outgoing edge $\left(v, v_{e}\right)$ corresponds to the choice of the edge $e$ in $\mathrm{G}^{\prime}$, and the cost of this edge in $\mathrm{G}^{\prime}$ is precisely the weight of the losing transition from $v_{e}$ in $\mathrm{G}$. Furthermore in G, all choices made from $V_{\diamond} \backslash V_{\diamond}^{\prime}$ induce no extra penalties (because the weight of all edges leaving those states is always 0 ). This implies that the cost of strategy $\sigma^{\prime}$ coincides with the penalty of multi-strategy $\sigma_{1}$.

\footnotetext{
${ }^{9}$ In the sense that each finite run is associated at most one edge.
} 


\section{B Proof of Lemma 5}

Lemma 5. Assume that we are given an optimal winning multi-strategy $\sigma$. We can effectively construct in polynomial time a memoryless multi-strategy $\sigma^{\prime}$, which is winning and optimal.

Proof. Assume that $W$ is the set of winning states. Let $\mathcal{T}$ be the forest representing the multi-strategy $\sigma$ (its set of roots is $W$ ). Since $\sigma$ is winning from every state in $W$, all branches of the forest are finite. For every node $t$ of $\mathcal{T}$, we define $\gamma_{\mathcal{T}}(t)$ as the residual penalty of $\sigma$ after prefix $t$. Formally, $\gamma_{\mathcal{T}}(t)=$ penalty $(\sigma, t)$. Obviously, for all $v \in V$, we have penalty $(\sigma, v)=\gamma_{\mathcal{T}}(v)$.

We will consider a measure $\mu_{\mathcal{T}}$ on the set of nodes of the forest $\mathcal{T}$ as follows: if $t$ is a node of $\mathcal{T}$, we let $\mu_{\mathcal{T}}(t)=\left(\gamma_{\mathcal{T}}(t)\right.$, height $\left.t_{\mathcal{T}}(t)\right)$.

We say that no memory is required for state $v$ in $\mathcal{T}$ if, for every two nodes $t$ and $t^{\prime}$ that are occurrences of $v$, the subtree of $\mathcal{T}$ rooted at $t$ and the subtree of $\mathcal{T}$ rooted at $t^{\prime}$ are identical. Note that in that case, $\mu_{\mathcal{T}}(t)=\mu_{\mathcal{T}}\left(t^{\prime}\right)$.

For every $0 \leq i \leq|V|$, we (inductively) build in polynomial time a forest $\mathcal{T}^{i}$ and a set $M_{i} \subseteq V$ containing $i$ elements, such that:

(a) $\mathcal{T}^{i}$ represents an optimal winning multi-strategy from all the states of $V$;

(b) for every $v \in M_{i}$, no memory is required for $v$ in $\mathcal{T}^{i}$, and for every node $t^{\prime}$ which is a descendant of some node that is an occurrence of $v$, letting $v^{\prime}=$ last $\left(t^{\prime}\right)$, it holds $v^{\prime} \in M_{i}$.

Intuitively, each $\mathcal{T}^{i}$ will be the forest of a winning optimal multi-strategy $\sigma_{i}$, and each $M_{i}$ will be a set of states from which $\sigma_{i}$ is memoryless $\left(i . e ., \sigma_{i}\right.$ is memoryless from the states in $M_{i}$, and from the states that occur in the outcomes from these states).

For $i=0$, it suffices to define $\mathcal{T}^{0}$ as the forest $\mathcal{T}$, and $M_{0}=\varnothing$. We assume we have already constructed the forest $\mathcal{T}^{i}=\left(T^{i}, V\right)$, and a corresponding set $M_{i}$. We pick a state $v_{i} \in V \backslash M_{i}$ such that there is an occurrence $t_{i} \in T^{i}$ of $v_{i}$ with

$$
\mu_{\mathcal{T}^{i}}\left(t_{i}\right)=\min \left\{\mu_{\mathcal{T}^{i}}(t) \mid t \text { is an occurrence of some } v \in V \backslash M_{i} \text { in } \mathcal{T}^{i}\right\} .
$$

Notice that each node $t$ in the subtree rooted at $t_{i}$ (in the forest $\mathcal{T}^{i}$ ) is an occurrence of a state of $M_{i}$. Indeed, if this were not the case, there would be a node $t_{i}^{\prime}$, which is a descendant of $t_{i}$, and which would then be such that $\gamma_{\mathcal{T}^{i}}\left(t_{i}^{\prime}\right) \leq \gamma_{\mathcal{T}^{i}}\left(t_{i}\right)$ and height ${\overline{\mathcal{T}^{i}}}\left(t_{i}^{\prime}\right)<$ height $_{\mathcal{T}^{i}}\left(t_{i}\right)$, contradicting the choice of $t_{i}$. In particular, there is no occurrence of $v_{i}$ in the subtree of $\mathcal{T}^{i}$ rooted at $t_{i}$.

The forest $\mathcal{T}^{i+1}$ is defined from the forest $\mathcal{T}^{i}$ by replacing every subtree rooted at an occurrence of $v_{i}$ with the subtree of $\mathcal{T}^{i}$ rooted at $t_{i}$. We define $M_{i+1}=M_{i} \cup\left\{v_{i}\right\}$ (which then has $i+1$ elements). It remains to check that all required conditions are satisfied. Clearly enough, since (by induction hypothesis) $\mathcal{T}^{i}$ represents a multi-strategy from all the states of $V$, this is also the case of $\mathcal{T}^{i+1}$. Pick an occurrence $t \in T^{i}$ of $v_{i}$ such that $v_{i}$ does not occur in any prefix of $t$. Then 
$t \in \mathcal{T}^{i+1}$. By definition of $t_{i}$, we obviously have that $\gamma_{\mathcal{T}^{i}}(t) \geq \gamma_{\mathcal{T}^{i}}\left(t_{i}\right)$. On the other hand, we also have that $\gamma_{\mathcal{T}^{i+1}}(t)=\gamma_{\mathcal{T}^{i}}\left(t_{i}\right) \leq \gamma_{\mathcal{T}^{i}}(t)$. These constraints propagate to all prefixes of $t$ in $\mathcal{T}^{i+1}$, hence the multi-strategy represented by $\mathcal{T}^{i+1}$ is optimal (from every state of $V$ ). This concludes the proof of condition $(a)$. By construction, no memory is required for state $v_{i}$ in $\mathcal{T}^{i+1}$. Assume that for some $v \in M_{i}$, there are two different subtrees of $\mathcal{T}^{i+1}$ rooted at an occurrence of $v$. It means that at least one of the subtrees has been changed by the substitution, hence that an occurrence of $v_{i}$ was in the subtree rooted at $t$. This contradicts condition (b) satisfied by $\mathcal{T}^{i}$ since $v_{i} \notin M_{i}$. Hence, the condition (b) is satisfied by $\mathcal{T}^{i+1}$.

The forest $\mathcal{T}^{|V|}$ represents a multi-strategy $\sigma^{\prime}$ which is memoryless, optimal and winning from every state of the game.

\section{Proof of Lemmas 6 and 7}

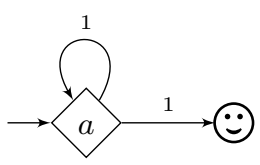

Fig. 6. No optimal discounted multi-strategy

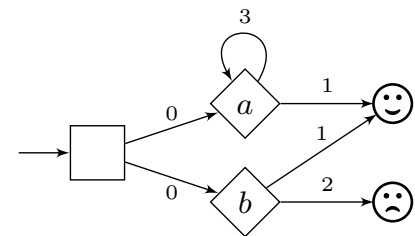

Fig. 7. No memoryless optimal discounted multi-strategy

Lemma 6. There exists weighted games for which there is no optimal winning multi-strategy under discounted penalties.

Proof. Figure 6 shows such a game. There is only one non terminal state and a multi-strategy for this game consists in choosing at each step whether to block the loop, the transition to the terminal state or none, with respective cost one, one and zero. In this game there is no multi-strategy with cost zero, since blocking nothing allows an infinite run staying in state $a$ forever. On the other hand a multi-strategy that blocks nothing the $n$ first steps and then blocks the loop has cost $\lambda^{n}$. Hence, for every $\varepsilon>0$, it is easy to design a $\varepsilon$-optimal discounted multi-strategy.

Lemma 7. There are weighted games under discounted penalties for which there exist a memoryful optimal winning multi-strategy but no memoryless one.

Proof. Figure 7 shows such a game. The only memoryless winning multi-strategy for this game blocks the losing transition and the loop and has penalty $3 \lambda$. Any winning multi-strategy has a penalty of at least $2 \lambda$ (the penalty of blocking the losing transition), and if we take a multi-strategy that blocks the loop only after $n$ steps with $3 \lambda^{n} \leq 2 \lambda$ (such a finite $n$ exists since $\lambda<1$ ) we get an optimal winning multi-strategy (which is obviously not memoryless). 


\section{Proof of Theorem 8}

Theorem 8. The optimal discounted-penalty problem is in NP $\cap$ coNP, and is PTIME-hard.

Proof. We let $\mathrm{G}=\left(V_{\square}, V_{\diamond}, E\right.$, weight $)$ be a finite weighted game, and let $c \in \mathbb{Q}$. Applying the transformation of Lemma 2 to the discounted case, we get an exponential-size weighted game $\mathrm{G}^{\prime}=\left(V_{\square}^{\prime}, V_{\diamond}^{\prime}, E^{\prime}\right.$, weight $\left.{ }^{\prime}\right)$ with $V_{\square} \subseteq V_{\square}^{\prime}$ and $V_{\diamond}=V_{\diamond}^{\prime}$ such that for every $v \in V_{\square} \cup V_{\diamond}$, Player $\diamond$ has a winning multi-strategy from $v$ in $\mathrm{G}$ with discounted penalty no more than $c$ (for discount $\lambda$ ) iff Player $\diamond$ has a winning strategy from $v$ in $\mathrm{G}^{\prime}$ with discounted cost no more than $c$ (for discount $\sqrt{\lambda}$ ). The game $G^{\prime}$ is memoryless determined [ZP96], which means that for every $c \in \mathbb{Q}$, for every state $v \in V_{\square}^{\prime} \cup V_{\diamond}^{\prime}$, either Player $\diamond$ has a memoryless strategy $\sigma_{\diamond}$ with $\operatorname{cost}^{\sqrt{\lambda}}\left(\sigma_{\diamond}, v\right) \leq c$, or Player $\square$ has a memoryless strategy $\sigma_{\square}$ with cost ${ }^{\sqrt{\lambda}}\left(\sigma_{\square}, v\right)>c$.

The NP algorithm is then as follows: guess a memoryless strategy $\sigma_{\diamond}$ for Player $\diamond$, i.e. for every $v \in V_{\diamond}$ guess a subset $F \subseteq v E$ and set $\sigma_{\diamond}(v)=(v, F)$. The game $\mathrm{G}^{\prime}$ where we have removed parts not allowed by $\sigma_{\diamond}$ has polynomial size and can be constructed from $G$ without first constructing $G^{\prime}$. In the resulting graph, from $v$, it is easy to compute in polynomial time the maximal discounted cost, which corresponds to $\operatorname{cost}^{\sqrt{\lambda}}\left(\sigma_{\diamond}, v\right)$. Thus we can decide in polynomial time whether $\operatorname{cost}^{\sqrt{\lambda}}\left(\sigma_{\diamond}, v\right) \leq c$.

Conversely, the coNP algorithm consists in guessing a strategy for Player $\square$ with cost larger than $c$. However, Player $\square$ controls exponentially many states in $\mathrm{G}^{\prime}$, and her strategy cannot be guessed in polynomial time. We have to first define a succinct encoding of memoryless optimal strategies of Player $\square$.

Applying [Jur98], we know that an optimal memoryless strategy $\sigma_{\square}$ can be computed, that is is characterized by values $v a l^{\sqrt{\lambda}}(v)=\operatorname{cost}^{\sqrt{\lambda}}\left(\sigma_{\square}, v\right)\left(\right.$ for $\left.v \in V^{\prime}\right)$ satisfying the following optimality equations:

$$
\begin{cases}v_{a l}{ }^{\sqrt{\lambda}}(v)=\min _{(v, F) \in V_{0}^{\prime}}\left(\text { weight }^{\prime}(v,(v, F))+\sqrt{\lambda} \cdot v a l^{\sqrt{\lambda}}(v, F)\right) & \text { if } v \in V_{\diamond} \\ v a l^{\sqrt{\lambda}}(v)=\operatorname{weight}^{\prime}(v,(v, v E))+\sqrt{\lambda} \cdot v a l^{\sqrt{\lambda}}(v, v E) & \text { if } v \in V_{\square} \\ v a l^{\sqrt{\lambda}}(\odot)=0 & \text { if }(v, F) \in V_{\square}^{\prime} \backslash V_{\square} \\ v a l^{\sqrt{\lambda}}(v, F)=\max _{e=\left(v, v^{\prime}\right) \in F}\left(\text { weight }^{\prime}\left((v, F), v^{\prime}\right)+\sqrt{\lambda} \cdot v_{a l}{ }^{\sqrt{\lambda}}\left(v^{\prime}\right)\right)\end{cases}
$$

By construction of $\mathrm{G}^{\prime}$, we can rewrite these equations as follows:

$$
\begin{cases}v_{a l}{ }^{\sqrt{\lambda}}(v)=\min _{F \subseteq v E}\left(\sum_{e \in v E \backslash F} \text { weight }(e)+\sqrt{\lambda} \cdot v a l^{\sqrt{\lambda}}(v, F)\right) & \text { if } v \in V_{\diamond} \\ v a l^{\sqrt{\lambda}}(v)=\sqrt{\lambda} \cdot v a l^{\sqrt{\lambda}}(v, v E) & \text { if } v \in V_{\square} \\ v a l^{\sqrt{\lambda}}(\odot)=0 & \text { if }(v, F) \in V_{\square}^{\prime} \backslash V_{\square} \\ v a l^{\sqrt{\lambda}}(v, F)=\max _{e=\left(v, v^{\prime}\right) \in F} \sqrt{\lambda} \cdot v_{a l}{ }^{\sqrt{\lambda}}\left(v^{\prime}\right) & \end{cases}
$$


Furthermore the strategy $\sigma_{\square}$ can be defined by:

$$
\begin{cases}\sigma_{\square}(v)=(v, v E) & \text { if } v \in V_{\square} \\ \sigma_{\square}((v, F))=\left((v, F), v^{\prime}\right) & \text { if }(v, F) \in V_{\square}^{\prime} \backslash V_{\square} \text { and } \\ & v l^{\sqrt{\lambda}}\left(v^{\prime}\right)=\max _{e=\left(v, v^{\prime \prime}\right) \in F} v a l^{\sqrt{\lambda}}\left(v^{\prime \prime}\right)\end{cases}
$$

In particular, we can define a total order $\prec$ (which we call a preference order) on states such that $v \prec v^{\prime}$ if $v a l^{\sqrt{\lambda}}(v) \geq v a l^{\sqrt{\lambda}}\left(v^{\prime}\right)$. For any $F \subseteq v E$, we define $v_{\prec}^{F}$ as the smallest element w.r.t. order $\prec \operatorname{such}$ that $\left(v, v_{\prec}^{F}\right) \in F$. We can then fix the strategy $\sigma_{\square}^{\prec}$ as follows:

$$
\begin{cases}\sigma_{\square}^{\prec}(v)=(v, v E) & \text { if } v \in V_{\square} \\ \sigma_{\square}^{\prec}((v, F))=\left((v, F), v_{\prec}^{F}\right) & \text { if } v \in V_{\square}^{\prime} \backslash V_{\square}\end{cases}
$$

The strategy $\sigma_{\square}^{\prec}$ is memoryless optimal for Player $\square$ and follows the preference order $\prec$, which means that if $v_{\prec}^{F}=v_{\prec}^{F^{\prime}}$, then $\operatorname{target}\left(\sigma^{\prec}((v, F))\right)=$ $\operatorname{target}\left(\sigma^{\prec}\left(\left(v, F^{\prime}\right)\right)\right)$. And this proves that we can restrict strategies for Player $\square$ in $G^{\prime}$ to memoryless strategies with a preference order on states. The main advantage of these strategies is that they have polynomial size.

The coNP algorithm proceeds as follows: guess a preference order $\prec$ on states of $\mathrm{G}$. We want to compute $\operatorname{cost}^{\lambda}\left(\sigma_{\square}^{\prec}, v\right)$ in $\mathrm{G}^{\prime}$ in polynomial time (thus without explicitely constructing $G^{\prime}$ ). To that aim, we construct a weighted game $G^{\prime \prime}$ whose discounted cost coincides with $\operatorname{cost}^{\lambda}\left(\sigma_{\square}^{\prec}, v\right)$. The game $\mathrm{G}^{\prime \prime}=\left(V_{\square}^{\prime \prime}, V_{\diamond}^{\prime \prime}, E^{\prime \prime}\right.$, weight $\left.{ }^{\prime \prime}\right)$ is defined as:

$-V_{\square}^{\prime \prime}=V_{\square} \cup\left\{\left(v, v_{\prec}^{F}\right) \mid F \subseteq v E\right.$ and $\left.(v, F) \in V_{\square}^{\prime}\right\}$ and $V_{\diamond}^{\prime \prime}=V_{\diamond}$; notice that, from the definition of $v_{\prec}^{F}$, there are only polynomially many states.

- the set $E^{\prime \prime}$ is made of:

- $\left(v,\left(v, v_{\prec}^{F}\right)\right) \in E^{\prime \prime}$ if $(v,(v, F)) \in E^{\prime}$;

- $\left(\left(v, v_{\prec}^{F}\right), v_{\prec}^{F}\right) \in E^{\prime \prime}$.

- the weight function is defined as:

- weight $^{\prime \prime}\left(v,\left(v, v_{\prec}^{F}\right)\right)=\min _{F^{\prime} \subseteq v E \text { s.t. } v_{\prec}^{F^{\prime}}=v_{\prec}^{F}}$ weight $^{\prime}(v,(v, F))$

- weight $^{\prime \prime}\left(\left(v, v_{\prec}^{F}\right), v_{\prec}^{F}\right)=0$

Then it is not difficult to realise that

$$
\text { weight }^{\prime \prime}\left(v,\left(v, v_{\prec}^{F}\right)\right)=\sum_{v^{\prime} \prec v_{\prec}^{F} \text { s.t. }\left(v, v^{\prime}\right) \in v E} \operatorname{weight}\left(v, v^{\prime}\right)
$$


Indeed, we restrict the order $\prec$ to $\left\{v^{\prime} \mid\left(v, v^{\prime}\right) \in v E\right\}=\left\{v_{1}, \cdots, v_{p}\right\}$, assuming $v_{1} \prec v_{2} \cdots \prec v_{p}$. Now, we can write:

$$
\begin{aligned}
\text { weight }^{\prime \prime}\left(v,\left(v, v_{i}\right)\right) & =\min _{F \subseteq v E \text { s.t. } v_{\prec}^{F}=v_{i}} \operatorname{weight}^{\prime}(v,(v, F)) \\
& =\min _{F \subseteq v E \text { s.t. } v_{1}, \cdots, v_{i-1} \notin F} \text { and } v_{i} \in F \\
& =\operatorname{weight}_{F \subseteq v E \text { s.t. } v_{1}, \cdots, v_{i-1} \notin F} \text { and } v_{i} \in F \\
& \left.\min _{e \in v E \backslash F} \operatorname{weight}(e)\right) \\
& =\sum_{j=1}^{i-1} \operatorname{weight}\left(v, v_{j}\right) \quad\left(\text { taking } F=v E \backslash\left\{\left(v, v_{j}\right) \mid 1 \leq j<i\right\}\right)
\end{aligned}
$$

Thus the game $G^{\prime \prime}$ can be computed in polynomial time. Furthermore all states in $V_{\square}^{\prime \prime}$ have at most one successor, this is thus not a game, but a graph in which we can easily compute in polynomial time the discounted cost. It remains to prove that the discounted cost in $\mathrm{G}^{\prime \prime}$ from $v$ (denoted $\operatorname{cost}_{\mathrm{G}^{\prime \prime}}^{\sqrt{\lambda}}(v)$ ) coincides with $\operatorname{cost}_{G^{\prime}}^{\sqrt{\lambda}}\left(\sigma_{\square}^{\prec}, v\right)$. We first notice that any run in $\mathrm{G}^{\prime \prime}$ can be seen as a run in $\mathrm{G}^{\prime}$ under strategy $\sigma_{\square}^{\prec}$, and the (discounted) costs coincide. Thus, $\operatorname{cost}_{\mathrm{G}^{\prime \prime}}^{\sqrt[\sqrt{\lambda}]{ }}(v) \geq$ $\operatorname{cost}_{\mathrm{G}^{\prime}}^{\sqrt{\lambda}}\left(\sigma_{\square}^{\prec}, v\right)$.

Let $\varrho$ be a run generated by $\sigma_{\square}^{\prec}$ from $v$ in $\mathrm{G}^{\prime}$ : we have $\varrho=v_{0}\left(v_{0}, F_{0}\right) v_{1}\left(v_{1}, F_{1}\right) \ldots$ with $\left(v_{i}, F_{i}\right)=\sigma_{\square}^{\prec}\left(v_{i}\right)$ if $v_{i} \in V_{\square}$ and $v_{i+1}=v_{i} \prec$ in any case. The run $\widetilde{\varrho}$ defined as $v_{0}\left(v_{0}, \widetilde{F_{0}}\right) v_{1}\left(v_{1}, \widetilde{F_{1}}\right) \cdots$ where $\widetilde{F}_{i}=F_{i}$ if $v_{i} \in V_{\square}$, and $\widetilde{F}_{i}$ is the largest subset of $v_{i} E$ so that $v_{i \prec} \widetilde{F}_{i}=v_{i}$. The run $\widetilde{F_{i}}$ is also generated by $\sigma_{\square}^{\prec}$, and its discounted is no more than that of $\varrho$. Furthermore this run can be played in $\mathrm{G}^{\prime \prime}$, hence we get the converse inequality: $\operatorname{cost}_{\mathrm{G}^{\prime \prime}}^{\sqrt{\lambda}}(v) \leq \operatorname{cost}_{\mathrm{G}^{\prime}}^{\sqrt{\lambda}}\left(\sigma_{\square}^{\prec}, v\right)$, hence equality holds.

Thus, $\operatorname{cost}_{G^{\prime}}^{\sqrt{\lambda}}\left(\sigma_{\square}^{\prec}, v\right)$ can be computed in polynomial time, and we can decide whether $\operatorname{cost}_{\mathrm{G}^{\prime}}^{\sqrt{\lambda}}\left(\sigma_{\square}^{\prec}, v\right)>c$ in polynomial time. 